\title{
Instilling Confidence in First Aid and CPR Training: Alternatives to Traditional Methods
}

Loriann Hynes \& Mike Recine

Traditional CPR and various levels of First Aid education include the use of basic cardiopulmonary resuscitation (CPR) manikins to teach participants the rudimentary skills of chest compressions and ventilations as these cannot be safely conducted on classroom participants. While this form of teaching is widely used, it is limited in its ability to provide effective feedback for learners relating to their ability to recognize emergency conditions and develop confidence in the efficacy of their actions.

\section{Using simulation manikins}

Influences within the affective domain of learning (those that relate to feelings, appreciations, values, motivations and attitudes) can be challenging for educators to convey. With the emerging technologies in human patient simulation manikins, valuable feedback is available that has been shown to improve student confidence in their skills as well as satisfaction with learning (Davis, Storjohann, Spiegel, Beiber \& Barletta, 2013). Different levels of simulation manikins can be used to create effective training environments where learners can assess "patient" signs and symptoms that include increased heart rate, decreased blood pressure, pupil changes, and severe bleeding. which cannot be role-played using classroom participants, and consequently improve the learning experience.

Simulation environments can also afford the opportunity for learners to physically execute the appropriate treatment and the critical thinking necessary to respond to emergency situations without the risk of real-life consequences of errors in judgment. This in turn builds confidence in learners (Miller, Macpherson \& Hynes, 2018) which can contribute to their ability to respond to emergency conditions in their daily lives and work.

\section{Implications}

Cost can be a prohibiting factor in First Aid/CPR training, however, there are a variety of manikins and training feedback mechanisms available to fit most budgets. It is the manner in which the feedback is utilized that has the greatest impact on learner development and confidence. As clinical educators, we have found repeated exposure to this form of training provides learners with the opportunity to practice communication, 
Conference Abstract

International First Aid Education Conference, 22-25 April 2018

critical thinking, clinical decision making and technical skills in a safe but realistic environment. (Wayne et al., 2008 and Palmer, Edwards \& Racchini, 2014).

\section{Conference activity}

In an interactive session, participants used a pre-test questionnaire to self-evaluate their perceived ability to perform CPR effectively (see supplemental file online). They then performed CPR sequences of treatment using three different types of manikin feedback. Feedback can range from low to high, based on the manikin's “fidelity" or capacity to provide learner feedback (Maran \& Glavin, 2003). Participants started with low fidelity (Laerdal Little Anne torso), then low fidelity with the Laerdal CPR meter and completed the exercise with a mid-fidelity Laerdal Resusci Anne QCPR manikin, all of which provided real-time feedback of their performance. After completing the three methods of CPR "training", participants completed the second part of the questionnaire where they re-evaluated their perceived CPR capabilities.

Participants were asked to reflect on the varying levels of feedback regarding skill performance and the benefits to their learning. Participants eagerly shared with one another the different types of feedback the manikins provided and how it can be used not only to enhance participants' skills but also instructors' abilities to teach life-saving skills. More comprehensive feedback was highly valued for more advanced skill sets, while less was considered valuable for first time lay person learners. The discussion evolved to how the varying levels of simulation could be used across first aid courses to different audiences, depending on the goals of the course.

\section{References}

Davis, L.E., Storjohann, T.A., Spiegel, J.J, Beiber, K.M., Barletta, J.F. (2013). High-fidelity simulation for advanced cardiac life support training. Am J Pharm Educ. 77(3):1-7.

Maran, N.J, Glavin, R.J. (2003). Low- to high-fidelity simulation - a continuum of medical education? Medical Education. 37(S1):22-28.

Miller, M.B., Macpherson, A.K., Hynes, L.M., (2018). Athletic therapy students' perceptions of highfidelity manikin simulation: a pilot study. Ath/ Train Educ. J 13(2):158-167.

Palmer, E., Edwards, T., Racchini, J. (2014). High-fidelity simulation meets athletic training education: an innovative collaborative teaching project. Athl Train Educ J. 9(2):96-100.

Wayne, D.B., Didwania, A., Feinglass, J., Fudala, M.J., Barsuk, J.H., McGaghie, W.C. (2008). Simulation 377 based education improves quality of care during cardiac arrest team responses at an academic teaching hospital. Chest. 133:56-61. 